\title{
Erich Saling-The Father of Prenatal and Perinatal Medicine
}

\section{Dudenhausen JW*}

Professor, Department of Obstetrics and Gynecology, Weill Cornell Medical College, Sidra Medical and Research Center, Qatar Foundation, Doha, State of Qatar

The title of the book that Erich Saling is best known for is, translated literally and word for word, "The Child in the Field of Obstetrics" (Das Kind im Bereich der Geburtshilfe). Saling's choice of this particular name for his work is very apt since it reflects the leitmotif underlying his life as a physician and a scientist: making the world of the unborn child accessible to medical care and scientific research.

After having studied medicine in Jena and Berlin, Erich Saling pursued further professional training as an obstetrician and gynecologist from 1952 until 1958 at Städtische Frauenklinik Berlin-Neukölln, acquiring his doctoral degree in 1952 at the Freie Universität Berlin. In 1963 , as part of his post-doctoral qualification as an academic lecturer, he presented his thesis on "The Ratios of Blood Gas and the Acid-Base Balance of a Fetus in the Course of an Unimpaired Birth." Saling went on to become an associate professor in 1968 at the Freie Universität Berlin, was appointed as head of the newly formed Department of Obstetrics at the Frauenklinik Neukölln in 1976, and became University Professor of Perinatal Medicine at the Freie Universität Berlin in that same year. He retired in late 1990.

Essentially, obstetrics in Europe had been a manual, operative discipline up until the mid- $20^{\text {th }}$ century; patho-physiological or pathobiochemical aspects were rarely considered. The counseling provided to pregnant women was rudimentary at best. The focus, both in counseling and treating during labor at the time, was on the health of the mother, on preserving her life and preventing any damages to her health. The textbooks available at the time generally did not include a chapter on the fetus or the newborn infant. Following the publication of Saling's book, however, a very few scientists started to enter the research field of obstetrics, which had been the sphere primarily of midwives in Germany, with the objective of enabling a child's birth to follow a more conscious course, with better monitoring, "in the field of obstetrics."

Saling's scientific interest initially focused on the diagnostics of depressed newborn infants and their treatment. His first papers on infants in obstetrics were published in 1958 and 1959. In the fifty years of scientific work that followed, this leitmotif essentially remained the same: the diagnostics of intra-uterine oxygen deficiency and its consequences, avoidance of pre-term deliveries and of growth-restricted children, the management of the breech presentation. However, what makes his contribution to science so exceptional is not the multitude of ideas that Saling pursued, which would make the list cataloguing them very long-it is his discovery of the unborn child. By his work, by his effort to gain access, in scientific terms, to the unborn child, Saling played a strong part among those who turned the midwifery of old into the obstetrics we know today. While he was not alone in doing so, it certainly was he who determined the direction, the content and the speed of this process. The work he did was seminal for prenatal and perinatal medicine.

His ideas, such as that of taking a blood sample from the fetus during birth (this fetal blood sampling also being referred to as the "Saling Technique"), or the concept of amnioscopy, have become standard practice in clinical and scientific work. His first publications are among the most frequently cited works published in the field. The introduction of fetal blood sampling and the resulting opportunities for further analysis of physiological and patho-physiological factors was the major milestone of scientific progress in obstetrics. With his concept, Saling revolutionized obstetrics, and many followed his lead, while others first had to overcome a certain resistance in the first years.

Some of the ideas Saling developed and published in the 1970s, such as the "birth cap" or the "expression belt," were not developed further for clinical routine, perhaps because the technology of the time, the IT or materials did not allow them to be produced such that they could serve in clinical practice. Such and other concepts were "newly" invented by others at a later time, developed further, and deployed subsequently.

The groundbreaking achievements made by pioneers like Erich Saling, Roberto Caldeyro-Barcia, and Stanley James showed that it was possible, both to assess fetal well-being, and to actually provide treatment in utero. The international cooperation of these early days of pioneering innovation was demonstrated at the symposium "Effects of Labor on the Fetus and the Newborn," which was held from October 1-3, 1964 in Montevideo/Uruguay.

Today, the importance of effective antenatal care with particular emphasis on maternal hygiene and nutrition is an undisputed fact. The value of fetal monitoring, ultrasound and invasive methods is recognized, and they are used as part of standard antenatal care in industrial societies. Advancements in neonatal intensive care have greatly improved the chances of premature babies' long-term survival, as early as 23 weeks of gestational age. However, the major foci of perinatal medicine continue to be infection, cerebral maturation, and prevention and treatment of preterm labor-still hard both to predict and to treat effectively.

Saling himself regarded his commitment to science, which went above and beyond the pursuit of insights and knowledge, to be a form of advocating the profession of physician in public. Where he believed structures to be obsolete, colleagues to be following outdated concepts, or journalists to be worshipers of the gutter press, he would criticize while making constructive suggestions. The development of obstetrics to become a scientific discipline and an equivalent pillar on which gynecology is based was and continues to be a major objective of his efforts.

When the German Society of Perinatal Medicine (Deutsche Gesellschaft für Perinatale Medizin) was chartered, along with its subsidiary societies all over the world, and when the Journal of Perinatal

${ }^{*}$ Corresponding author: Dr. Dudenhausen JW, Professor, Department of Obstetrics and Gynecology, Weill Cornell Medical College, Deputy Chief Medical Officer, Sidra Medical and Research Center, Qatar Foundation, PO Box 26999, Doha, State of Qatar, E-mail: Joachim.Dudenhausen@charite.de

Received March 29, 2013; Accepted May 05, 2013; Published May 12, 2013

Citation: Dudenhausen JW (2013) Erich Saling-The Father of Prenatal and Perinatal Medicine. J Health Med Informat S11: 008. doi:10.4172/2157-7420.S11008

Copyright: (c) 2013 Dudenhausen JW, et al. This is an open-access article distributed under the terms of the Creative Commons Attribution License, which permits unrestricted use, distribution, and reproduction in any medium, provided the original author and source are credited. 
Medicine was established, these were offers made to pediatricians, inviting them to enter into discourse and cooperate at an international level in the interests of their child patients and for their benefit. This collaboration is now being practiced globally, with a true focus on the goals to be achieved, and without any friction among those involved.

The last 40 to 50 years have seen the founding of many national and international societies of perinatal medicine, including the World Association of Perinatal Medicine (WAPM). Scientists from the fields of obstetrics, neonatology, physiology, and neighboring disciplines came together in Barcelona 2005 and formed the International Academy of Perinatal Medicine (IAPM). This independent, international, scientific and academic non-profit institution with 30 permanent fellows from all over the world chaired by Erich Saling has made it its purpose to serve as a forum for the assessment and discussion of worldwide perinatal medicine, and owes its existence to the preparatory work done by the
World Association of Perinatal Medicine, The International Society of the Fetus as a Patient and the European Association of Perinatal Medicine. Erich Saling wish is to draw up guidelines focusing on practical topics such as the prevention of preterm births, while also intensifying and broadening the academic discussions of ethics and the impact of globalization on perinatal medicine.

Erich Saling's life is a series of successes. He has been honored and recognized by many. Scientific prizes, courses, and medical schools have been named for him.

I have had the privilege and honor to work together with Erich Saling as a resident, as a consultant, and as his deputy director. I have many things in my life to thank him for. To this day, our regular exchanges and discussions bear witness to the instruction I received from him at the start, and to our later cooperation. 